\title{
Super-regular breathers in nonlinear systems with self-steepening effect
}

\author{
Chong Liu ${ }^{1,2,3}$ and Nail Akhmediev ${ }^{2}$ \\ ${ }^{1}$ School of Physics, Northwest University, Xi'an 710127, China \\ ${ }^{2}$ Optical Sciences Group, Research School of Physics and Engineering, The Australian National University, Canberra, ACT 2600, Australia \\ ${ }^{3}$ Shaanxi Key Laboratory for Theoretical Physics Frontiers, Xi'an 710127, China
}

(Received 10 August 2019; published 3 December 2019)

\begin{abstract}
A family of super-regular (SR) breather solutions in systems with self-steepening effect and in the case of either normal or anomalous dispersion is derived analytically. Derivation is based on the Darboux transformation with a quadratic spectral parameter. In contrast to the SR breather solutions in $t$-symmetric systems such as the nonlinear Schrödinger equation, the new breathers found in the present work evolve asymmetrically even if started from symmetric initial conditions. The initial stage of this process is modulation instability. Numerical simulations confirm the excitation of the SR breathers when started from the approximate initial conditions leading at first to modulation instability. Our results offer the possibility of experimental observations of SR breather dynamics in systems with self-steepening effects, such as optical frequency-doubling crystals or magnetized plasmas.
\end{abstract}

DOI: 10.1103/PhysRevE.100.062201

\section{INTRODUCTION}

Waves oscillating on a plane-wave background, known as breathers, have received much attention in nonlinear science over the past decade [1-16]. They provide a basis for more complicated formations-nonlinear superpositions of breathers that appear in many nonlinear phenomena of physical importance such as rogue-wave events [10-13], breatherwave molecules [14], chess-board-like interference patterns [15], and modulation instability (MI), where small periodic modulation is additionally localized in transverse direction. One way of creating these types of initial conditions is using a nonlinear superposition of pairs of fundamental (quasiAkhmediev) breathers propagating at slightly tilted angles with opposite signs to the main direction of propagation. The resulting exact solutions in the form of symmetrically diverging modulated beams (regular breathers) have been named "super-regular (SR) breathers" by the authors of Ref. [16].

From a theoretical point of view, SR breathers are higherorder exact solutions of the nonlinear Schrödinger equation (NLSE) [16-18]. One of the ways to construct these exact solutions is to parameterize the spectral parameter of the associated Lax pair using the Jukowsky transform [19]. The existence of SR breathers, consisting of a symmetric pair of breathers, has been confirmed recently in an optical fiber and in a water-wave arrangement [18]. Experimental research tightly follows intense theoretical studies whose purpose is deeper understanding of the nature of SR breathers. So far, SR breathers have been studied in the NLSE hierarchy with various higher-order terms [20-22], in a resonant erbiumdoped fiber [23], and in a self-induced transparency media [24]. In these studies, some intricate SR breather properties have been demonstrated. For example, it was shown that the MI growth rate of SR breathers coincides with the absolute difference of group velocities of the pair of breathers involved in the solution [22]. Importantly, the role of SR breathers in rogue-wave formation has been revealed in Ref. [25]. The nonlinear stage of MI in formation of SR breathers has also been carefully studied $[26,27]$.

So far, all theoretical findings are limited to integrable dynamical systems with the associated Lax pairs and linear spectral parameters. These include SR breathers in the infinite NLSE hierarchy [22]. On the other hand, SR breathers in important classes of integrable systems admitting nonlinear spectral parameter in the spatial Lax operator remains completely unexplored. Among them, we can mention the WadatiKonno-Ichikawa (WKI) system [28] which admits quadratic spectral parameter in the corresponding spatial Lax operator. These type of systems with self-steepening terms have attracted considerable attention in both theory and experiment [29] as these terms are important in describing ultrashort pulses in femtosecond lasers.

Self-steepening of optical pulses is the result of the intensity-dependent group velocity [30]. Self-steepening naturally leads to asymmetric optical spectra. It also results in the chirped [31,32] or two-color walking [33] double-localized Peregrine rogue waves that are the limiting case of breathers with infinite period. On the other hand, SR breathers which are a special superposition of pairs of breathers remain unknown for systems with self-steepening. The related problem of MI growth rate for this case is also need to be solved. Another question remained to be answered is an excitation of SR breathers from initial conditions that are not exactly defined by the SR breather solutions but are sufficiently close to them. On the one hand, solving this latter problem will provide an answer to the problem of robustness of SR breathers. On the other hand, it will allow us to choose the initial conditions that are much closer to realistic experimental conditions.

In this paper, we address these questions by finding both analytically and numerically the SR breather solution in a nonlinear system with a single self-steepening term in the cases of either normal or anomalous dispersion. Such a system 
is governed by the following Chen-Lee-Liu equation (CLLE) [34]:

$$
i \frac{\partial \psi}{\partial z}+\sigma \frac{\partial^{2} \psi}{\partial t^{2}}+i|\psi|^{2} \frac{\partial \psi}{\partial t}=0
$$

where $\psi(z, t)$ is the wave envelope, $z$ is the propagation variable, while $t$ is retarded time in the frame moving with the wave group velocity. Coefficient $\sigma$ defines the sign of dispersion. It is positive $(\sigma=+1)$ when dispersion is anomalous and negative $(\sigma=-1)$ when it is normal. Equation (1) differs from the NLSE by the last term in the left-hand side. Namely, the cubic self-phase modulation term $|\psi|^{2} \psi$ is simply replaced by the term $i|\psi|^{2} \psi_{t}$ describing the effect of self-steepening. In experiments, it is a challenging task to separate the contributions of self-steepening and self-phase modulation effects. Despite these difficulties, the relative influence of these terms on ultrashort optical pulse propagation has been revealed in recent work [35] in a frequency-doubling crystal via the interplay of quadratic and cubic nonlinearities. This can also be done in quadratic nonlinear media [36].

An important point is that the CLLE (1) is integrable. It belongs to the WKI system [28]. In this generalized approach, the spatial operator of the associated Lax pair admits quadratic spectral parameter. The first-order breather solution for this case has been found in Ref. [37]. The bright soliton and dispersive shock waves have been studied in Refs. [38] and [39], respectively. The SR breather solution is given in the present work.

The CLLE is one of the simplest equations that includes a self-steepening term and that belongs to the WKI system. Another similar case is the Kaup-Newell equation [40], where the self-steepening term has a form, $\frac{\partial}{\partial t}\left(|\psi|^{2} \psi\right)$. The latter operator can be split:

$$
\frac{\partial}{\partial t}\left(|\psi|^{2} \psi\right)=\psi \frac{\partial}{\partial t}\left(|\psi|^{2}\right)+|\psi|^{2} \frac{\partial}{\partial t} \psi
$$

which means that there are other forms of equations with selfsteepening terms. Among them, we can mention the mixedtype NLSE [41], the Fokas-Lenells equation [42], the cubicquintic NLSE [43], and the higher-order CLLE extension [44]. All these equations are found to be integrable.

Finding SR breathers for these equations is still a challenge. Common features and differences between the SR breathers in these models will enrich our understanding of the MI phenomena influenced by self-steepening. Yet it is impossible to study SR breathers in these models all in one step. Thus here we make only the first step finding the SR breather for the CLLE. Due to the simplicity of the selfsteepening term in the CLLE (1), the study of the SR breathers for this model is of fundamental importance. Generalizing these results for other equations at later times will enrich our understanding of self-steepening effects in general.

The paper is organized as follows. The derivation of the exact breather solution is given in Sec. II. The SR breather with a reflection-symmetry-breaking dynamics is obtained in Sec. III. The relation between the MI and SR breather is demonstrated in Sec. IV. Section V contains our conclusions.

\section{EXACT BREATHER SOLUTION AND ITS SELF-STEEPENING}

Breathers in general and SR breathers in particular can be generated as a result of modulation instability. Periodic perturbation extends to infinity in the case of the standard MI, while it is localized in the case of SR breathers. Thus let us start our investigation with the linear stability analysis. Similarly to the NLSE, Eq. (1) has a plane-wave solution,

$$
\psi_{0}=a \exp (i \theta), \quad \theta=q t-\left(\sigma q^{2}+a^{2} q\right) z,
$$

where $a$ and $q$ are its amplitude and frequency, respectively. Adding a periodic perturbation

$$
\psi_{p}=\left[a+f_{+} e^{i(Q t+\omega z)}+f_{-}^{*} e^{-i\left(Q t+\omega^{*} z\right)}\right] e^{i \theta}
$$

with a frequency $\omega$, wave number $Q$ and small amplitudes $f_{+}, f_{-}^{*}$ and linearizing the solution around the plane wave, we obtain the dispersion relation

$$
\omega=a^{2} Q+2 \sigma q Q \pm Q \sqrt{2 a^{2} \sigma q+Q^{2}} .
$$

The plane wave (2) is modulationally unstable when $\operatorname{Im}\{\omega\} \neq$ 0 . This happens when $|Q|<a \sqrt{-2 \sigma q}$ (or $\sigma q<0$ ). The small initial modulation in this frequency range grows exponentially. The growth rate of this instability is given by $G=$ $|\operatorname{Im}\{\omega\}|$. The maximum growth rate $G_{m}=-\sigma q a^{2}$ is achieved when $|Q|=a \sqrt{-\sigma q}$.

We note that $q(\neq 0)$ and $\sigma$ should have opposite signs to ensure that $\operatorname{Im}\{\omega\} \neq 0$. This implies that the plane wave can be modulationally unstable in the cases of either normal or anomalous dispersion. With no loss of generality, we choose $\sigma=+1$. Then the two cases are covered by varying the sign of $q$.

The initial stage of evolution is described by the above equations. However, in order to have the full-scale evolution beyond the linear approach, we need exact solution that is valid at any $z$. In what follows, we construct exact SR breather solutions that can be considered as nonlinear continuation of the MI approximation.

In order to do that, we first represent Eq. (1) in the form of two linear equations with $2 \times 2$ matrix operators [45]:

$$
\boldsymbol{\Phi}_{t}=\mathbf{U} \boldsymbol{\Phi}, \quad \boldsymbol{\Phi}_{z}=\mathbf{V} \boldsymbol{\Phi},
$$

where $\boldsymbol{\Phi}=(R, S)^{\top}$ ( $\mathrm{T}$ means a matrix transpose $)$ and

$$
\begin{gathered}
\mathbf{U}=\left(\begin{array}{cc}
i \frac{\sigma}{4}|\psi|^{2}-i \lambda^{2} & \psi \lambda \\
-\sigma \lambda \psi^{*} & i \lambda^{2}-i \frac{\sigma}{4}|\psi|^{2}
\end{array}\right), \\
\mathbf{V}=\left(\begin{array}{cc}
v_{1} & v_{2} \\
v_{3} & -v_{1}
\end{array}\right),
\end{gathered}
$$

with

$$
\begin{aligned}
& v_{1}=-2 i \lambda^{4}+i \sigma|\psi|^{2} \lambda^{2}-\frac{1}{8} i|\psi|^{4}+\frac{\sigma}{4}\left(\psi \psi_{t}^{*}-\psi^{*} \psi_{t}\right), \\
& v_{2}=\left(i \psi_{t}-\frac{\sigma}{2}|\psi|^{2} \psi\right) \lambda+2 \lambda^{3} \psi, \\
& v_{3}=\left(i \sigma \psi_{t}^{*}+\frac{1}{2}|\psi|^{2} \psi^{*}\right) \lambda-2 \sigma \lambda^{3} \psi^{*} .
\end{aligned}
$$


Here $\lambda$ is the spectral parameter. The CLLE (1) follows from the compatibility condition

$$
\mathbf{U}_{z}-\mathbf{V}_{t}+[\mathbf{U}, \mathbf{V}]=0 .
$$

Breather solutions can be constructed using the Darboux transformations [46] and the seeding solution in the form of the plane-wave background (2). One can construct breather solutions by solving the Lax pair with an arbitrary complex spectral parameter $\lambda$, following the previous works $[11,12]$. SR breather solutions require two spectral parameters.

The Lax pair (6) can be written in the following form:

$$
\tilde{\mathbf{U}}=\left(\begin{array}{cc}
i \beta & a \lambda \\
-a \lambda & -i \beta
\end{array}\right), \quad \tilde{\mathbf{V}}=\left(\begin{array}{cc}
-i \beta \rho & -a \lambda \rho \\
a \lambda \rho & i \beta \rho
\end{array}\right),
$$

using a diagonal matrix $\mathbf{s}=\operatorname{diag}\left(e^{-i \theta / 2}, e^{i \theta / 2}\right)$ and the functions

$$
\begin{aligned}
& \beta\left(\lambda^{2}\right)=\frac{1}{4} a^{2}-\lambda^{2}-\frac{1}{2} q, \\
& \rho\left(\lambda^{2}\right)=\frac{1}{2} a^{2}-2 \lambda^{2}+q .
\end{aligned}
$$

As a result, the linear eigenvalue problem (6) reduces to that of Eq. (7) with the two eigenvalues given by

$$
\tau= \pm i f\left(\lambda^{2}\right), \quad f\left(\lambda^{2}\right)=\sqrt{\beta\left(\lambda^{2}\right)^{2}+a^{2} \lambda^{2}} .
$$

We remark here that if $\operatorname{Im}\left\{\tau^{2}\right\}=0$, and $\operatorname{Re}\left\{\tau^{2}\right\} \neq 0$, the corresponding solution describes either the Akhmediev breather (AB) [47] or the Kuznetsov-Ma soliton dynamics [48]. In the degenerate case $\tau=0$, the resulting solution is further reduced to the rational expression of the Peregrine rogue wave [49]. In the latter case, the spectral parameter satisfies

$$
\lambda^{2}=i \frac{a}{2} \sqrt{-2 q}-\frac{1}{4}\left(a^{2}+2 q\right) .
$$

This is a special value of $\lambda$ in the dynamics of fundamental nonlinear waves. In contrast to the case of the infinitely extended NLSE hierarchy [50] and to the previous work for the CLLE [37], we express this spectral parameter, Eq. (9), in the quadratic form.

One way of expressing the spectral parameter in finding the SR breather solution is the Jukowsky transform [19]. We start with the transform for the imaginary part of the quadratic spectral parameter (9):

$$
\lambda^{2}=i \frac{a}{4}\left(\xi+\frac{1}{\xi}\right) \sqrt{-2 q}-\frac{1}{4}\left(a^{2}+2 q\right) \equiv \mu^{\prime}+i v^{\prime},
$$

where $\xi=r \exp (i \alpha)$ with $r$ and $\alpha$ being the radius and angle of the polar coordinates in the sector defined by $r \geqslant 1, \alpha \in$ $(-\pi / 2, \pi / 2)$. Then the real $\mu^{\prime}$ and imaginary $\nu^{\prime}$ parts of $\lambda^{2}$ are given by

$$
\begin{gathered}
-\mu^{\prime}=\frac{a}{4}\left(r-\frac{1}{r}\right) \sqrt{-2 q} \sin \alpha+\frac{1}{4}\left(a^{2}+2 q\right), \\
v^{\prime}=\frac{a}{4}\left(r+\frac{1}{r}\right) \sqrt{-2 q} \cos \alpha .
\end{gathered}
$$

The convenience of the transformation (10) is in mapping of the spectral parameter to the plane in polar coordinates with two values of $\alpha$ with opposite signs. Interactions between two such breathers can potentially generate a SR breather. The phase of each breather is also relevant (see Sec. III).

To start with, let us first consider a single fundamental breather. Using the definition (10) of the spectral parameter through its quadratic value $\lambda \equiv \mu+i v=\sqrt{\lambda^{2}}$ and solving the associated Lax pair (6), as shown in the Appendix A, we obtain the first-order breather solution in complex form:

$$
\psi(z, t)=\left[\frac{G(z, t)+i H(z, t)}{D_{1}(z, t)+i D_{2}(z, t)}-1\right] \psi_{0}(z, t),
$$

where the expressions in the numerator $G, H$, and denominators $D_{1}$ and $D_{2}$ are real functions:

$$
\begin{gathered}
G=4 v^{\prime} K \cosh \gamma_{1}+\frac{2 v^{\prime}}{a} A \cos \kappa_{1}+2 D_{1}, \\
H=4 v^{\prime} K^{\prime} \sinh \gamma_{1}+\frac{2 v^{\prime}}{a} A^{\prime} \sin \kappa_{1}, \\
D_{1}=\mu A \cosh \gamma_{1}+2 a \mu K \cos \kappa_{1}, \\
D_{2}=v A^{\prime} \sinh \gamma_{1}-2 a v K^{\prime} \sin \kappa_{1}
\end{gathered}
$$

with

$$
\begin{aligned}
\gamma_{1} & =2 \gamma\left(t-V_{g} z\right), \quad \kappa_{1}=2 \kappa\left(t-V_{p} z\right)-\theta_{1}, \\
A & =a^{2}\left(v^{2}+\mu^{2}\right)+\left(\delta^{2}+\eta^{2}\right), \quad K=\mu \delta-v \eta, \\
A^{\prime} & =a^{2}\left(v^{2}+\mu^{2}\right)-\left(\delta^{2}+\eta^{2}\right), \quad K^{\prime}=v \delta+\mu \eta .
\end{aligned}
$$

Here $V_{g}, V_{p}$ are the group and phase velocities

$$
\begin{aligned}
& V_{g}=\frac{a^{2}}{2}+q-2 \mu^{\prime}+\frac{2}{\gamma} \kappa \nu^{\prime}, \\
& V_{p}=\frac{a^{2}}{2}+q-2 \mu^{\prime}-\frac{2}{\kappa} \gamma \nu^{\prime},
\end{aligned}
$$

with $\eta=\frac{a^{2}}{4}-\kappa-\mu^{\prime}-\frac{q}{2}, \delta=\gamma-v^{\prime}$, and

$$
\begin{aligned}
& \kappa=\frac{a}{4} \sqrt{-2 q}\left(r+\frac{1}{r}\right) \sin \alpha, \\
& \gamma=\frac{a}{4} \sqrt{-2 q}\left(r-\frac{1}{r}\right) \cos \alpha .
\end{aligned}
$$

The solution (13) depends on the plane-wave parameters $(a, q)$, the spectral parameter in polar coordinates $(r, \alpha)$, and the phase $\theta_{1}$. Similarly to the case of the NLSE breather solution [11], Eq. (13) describes the growth-decay cycle of periodic structure on top of the plane wave. This structure is propagating with the group velocity $V_{g}$ and the phase velocity $V_{p}$. At special values of the spectral parameter, the solution (13) reduces to Akhmediev breather $(r=1, \alpha \neq$ $0)$, Kuznetsov-Ma soliton $(r \neq 1, \alpha=0)$, or Peregrine rogue wave $(r=1, \alpha=0)$. In the remaining cases, the solution describes the general (Tajiri-Watanabe) breather [51].

In contrast to the previous cases [10-12]), the denominator $D_{1}+i D_{2}$ in Eq. (13), is left complex. This form can be easily transformed into more common form by simply multiplying both numerator and denominator by the complex conjugate expression $D_{1}-i D_{2}$.

The simplest SR breather is a nonlinear superposition of two quasi-ABs [16] with $r=1+\varepsilon(\varepsilon \ll 1), \alpha \neq 0$. In other words, interaction between two quasi-ABs with opposite $\alpha$ generates potentially a SR breather. As can be seen 

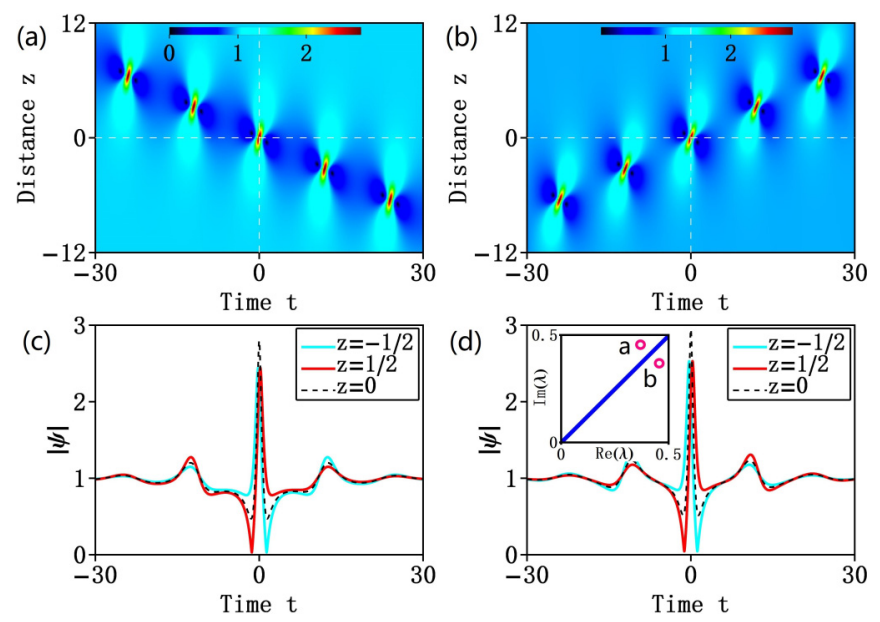

FIG. 1. Amplitude profiles of two quasi-Akhmediev breathers, described by Eq. (13), with values of $\alpha$ of opposite sign: (a) $\alpha=\pi / 6$ and (b) $\alpha=-\pi / 6$. The panels (c) and (d) show specific shape of functions in (a) and (b) at three distances: $z=-1 / 2, z=0$, and $z=$ $1 / 2$. The inset in (d) shows the location of the spectral parameters of breathers presented in (a) and (b) on the $(\operatorname{Re}(\lambda), \operatorname{Im}(\lambda))$ plane (red circles) as given by Eq. (10). The parameters used in calculation are $a=1, q=-0.5, r=1.15, \theta_{1}=0$.

from Eq. (18), these two quasi-ABs share the same width $[\sim 1 /(2 \gamma)]$ and modulation frequency $2 \kappa$ in $t$. The latter is approximated by $2 \kappa=a \sqrt{-2 q} \sin \alpha($ as $\varepsilon \ll 1)$, which is the same as that of $\mathrm{AB}$. However, due to the asymmetry relative to the transformation $t=>-t$, the maximal amplitudes $(|\psi|=$ $\left.2 v^{\prime} / \mu+a\right)$ of the two quasi-ABs are not exactly equal.

Figure 1 shows a particular case of two quasi-ABs propagating with opposite group velocities $\left(V_{g}\right)$ when $a^{2} / 2+q=$ 0 . The corresponding spectral parameters are symmetrically located relative to the line $\operatorname{Re}(\lambda)=\operatorname{Im}(\lambda)$. They are displayed in the inset of Fig. 1(d). Each period of the quasi-ABs has an amplitude peak and two side holes tilted relative to the $t$ axis. Transitions from hole to peak can be considered as shock waves, as can be seen from Figs. 1(c) and 1(d). This effect results from the self-steepening similar to that in the case of a single Gaussian or soliton-like pulse [30,35]. Clearly, in the breather case, formation of shock waves occurs with periodicity of the breather itself. Another remarkable feature of the two quasi-ABs in Fig. 1 is their asymmetry. This is the consequence of asymmetry in time transformation $t \rightarrow-t$ of the original equation.

\section{SR BREATHERS AND SYMMETRY BREAKING}

Nonlinear interaction between the two quasi-ABs presented above produces SR breathers. The phases $\theta_{1}, \theta_{2}$ play a significant role in their formation as they shift the wave profiles in each period of the quasi-ABs as can be seen from Fig. 2 below. In the previous works [16-18], SR breathers are generated with the phase relation $\theta_{1}+\theta_{2}=\pi$. This condition created specific symmetric pattern of interaction with relatively small amplitudes on the line $z=0$. With the selfsteepening term in the CLLE (1), we consider two possibilities: $\left\{\theta_{1}, \theta_{2}\right\}=\{0, \pi\}$ and $\left\{\theta_{1}, \theta_{2}\right\}=\{\pi, 0\}$. Other cases lead to irregular SR patterns with high amplitudes in the middle.
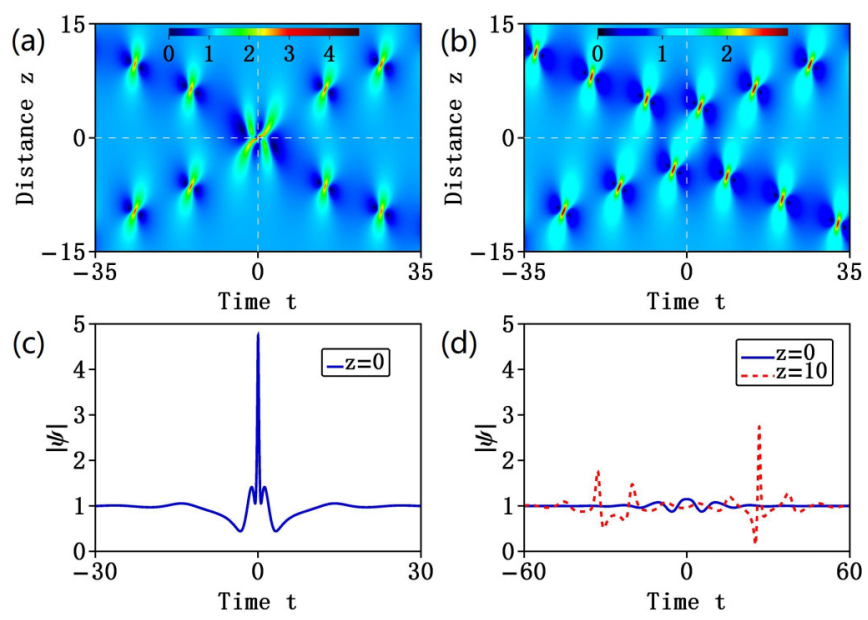

FIG. 2. Collision of two quasi-ABs propagating with opposite velocities. It is described by the exact solution in Appendix B, where (a) $\theta_{j}=0$ and (b) $\theta_{j}=\{0, \pi\}$. The panels (c) and (d) show the amplitude profiles of the cases (a) and (b), respectively, at $z=0$ (blue curve) and at $z=10$ [red curve in (d)]. Other parameters are the same as in Fig. 1.

These type of patterns cannot be considered as being produced due to the MI. The two patterns with the above phase relations are shown in Fig. 2. They are produced using the exact SR breather solution given in Appendix B.

Figure 2 shows the interaction pattern of two quasi-ABs in the cases when the two individual components shown in Fig. 1 are in phase $\left[\theta_{1}=0, \theta_{2}=0\right.$, Fig. $\left.1(\mathrm{a})\right]$ or out of phase $\left[\theta_{1}=\right.$ $0, \theta_{2}=\pi$, Fig. 1(b)]. In the first case, the field profile at the origin reaches its maximal value as shown in Fig. 2(c). In the second case, the central amplitude is relatively small as shown in Fig. 2(d). The central feature in the first case is the secondorder rogue wave [10-13]. In the second case, the wave profile at $z=0$ can serve as the initial condition for excitation of SR breather.

The choice of phase difference between the two components in Fig. 2, to some extent, is similar to the choice of phases in the collision dynamics of the NLSE bright solitons [52,53]. However, the breather collision occurs on top of a plane wave while ordinary solitons collide on a zero background. Clearly, SR breather does require plane-wave background in order to start with MI.

SR breathers are of interest for physics as one of the ways to observe MI dynamics with localized initial perturbation. This initial condition is given by the SR solution $\psi(0, t)$ at $z=0$. Just as in the NLSE case, the initial conditions for generation of SR breathers are even perturbations in $t$ [Fig. 2(d)]. However, noneven perturbations can also grow into non symmetric breathers. They are not super-regular in the same way as in Ref. [16] but strongly related to MI [27].

The new feature of the dynamics in the present case is the asymmetry accumulated when $z$ is increasing. This asymmetry is clearly seen when looking at the red dashed curve in Fig. 2(d). This asymmetry is absent in the SR breather evolution of the standard NLSE [16-18]. Here the symmetric initial condition evolves into a pair of quasi-ABs with 

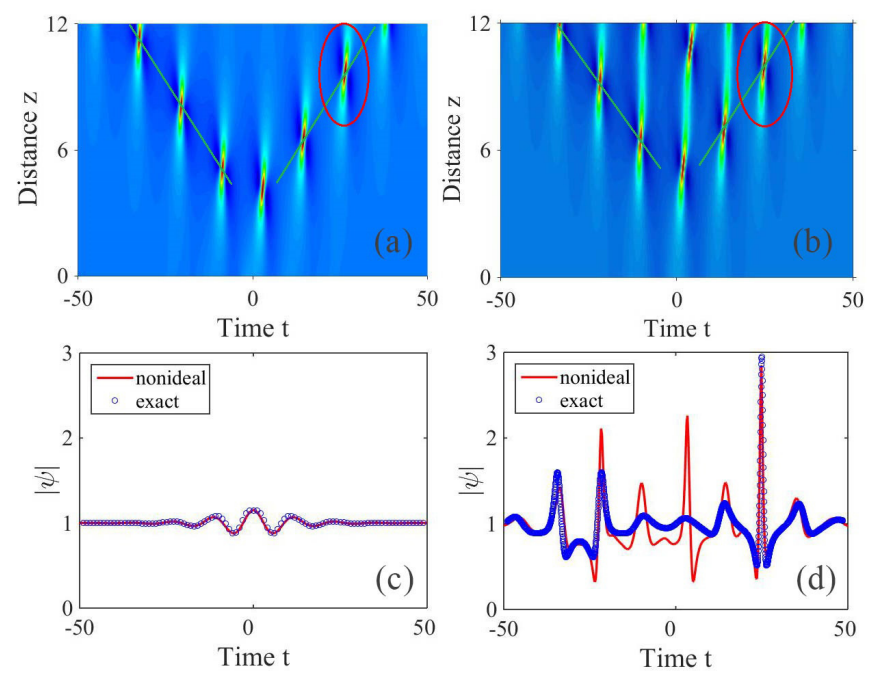

FIG. 3. The results of numerical simulations of the SR breather propagation that starts from (a) exact initial conditions and (b) approximate ones (23) [here $\rho=0.15, \kappa$ and $\gamma$ are given by Eqs. (21) and (22), respectively]. Panel (c) shows the amplitude profiles at $z=0$ for simulations shown in (a) and (b). Panel (d) shows the amplitude profiles at the values of $z$ located in the middle of red ellipses in (a) and (b) where the amplitudes of the wave fields are maximal. The green lines in (a) and (b) are positioned to show the group velocities of the two breathers, given by Eq. (19). Parameters of simulations are the same as in Fig. 2.

different wave profiles. This case clearly shows the influence of the self-steepening phenomenon on the wave propagation.

An explicit expression for the initial condition $\psi(0, t)$ in the CLLE (1) is complicated and is not given here. Being complicated, it can still be used as initial condition in numerical simulations. Another possibility is to use approximations which are relatively close to the exact expression. As another advantage, simple approximate initial conditions can be used for experimental observations. In order to demonstrate this possibility, we have solved the CLLE numerically using the split-step Fourier method. As the initial condition, we used the following approximation:

$$
\psi=[a+\rho \operatorname{sech}(2 \gamma t) \cos (2 \kappa t)] e^{i \theta},
$$

where $\rho$ is a small perturbation amplitude $(\rho \ll 1)$. We take it to be real in order to fit the exact initial condition [see Fig. 3(c)]. This is different from the NLSE case, where the SR breather starts with an initial condition in purely imaginary form [16]. Besides, real values are easier to implement in experiments [54]. For comparison, we made similar numerical simulations starting with the exact initial conditions given by the SR breather solution $\psi(0, t)$ at $z=0$. We accurately approximated the frequency $2 \kappa$ and the width $2 \gamma$ of the perturbation in Eq. (23) to the values given by the exact solution.

Figure 3 shows the results of numerical simulations that started with the exact initial conditions [Fig. 3(a)] as well as the nonideal initial condition, Eq. (23) [Fig. 3(b)]. Remarkably, SR breathers are well reproduced in each case. Moreover, SR breathers propagate with the group velocities that coincide with the exact results given by Eq. (19). Despite the more complex oscillating structure of the MI pattern in the case of nonideal initial conditions (23), the structures along the edges of the triangle given by the group velocities are roughly the same in the two cases. This can be clearly seen from Fig. 3(d), where the edges of the two profiles shown in red and blue curves nearly overlap. Deviation from the exact initial conditions causes the appearance of additional peaks inside the triangular area.

\section{SR BREATHERS AND MODULATION INSTABILITY}

Akhmediev breathers are tightly related to modulation instability [47]. As a consequence, the SR breathers also describe developed stage of MI although this connection is not as simple as in a single breather case [47]. Such a connection has been partially analyzed for the infinite NLSE hierarchy [22] as well as for the coupled NLSE-Maxwell-Bloch model [23]. Here we make one more step forward toward understanding this issue.

For an elementary SR breather formed by a pair of quasiABs with initial frequencies $2 \kappa$, the common MI growth rate is given by

$$
G=\left|q a^{2} \sin 2 \alpha\right|
$$

However, this MI growth rate is not the same as the growth rate along $z$ provided by each quasi-AB $2 \gamma\left|V_{g j}\right|$. This implies that there is a new physics in the process of the MI growth driven by the SR breathers. In order to see this, we define the absolute value of the difference between the two group velocities:

$$
\Delta V_{g} \equiv\left|V_{g 1}-V_{g 2}\right|=2 a \sqrt{-2 q}|\sin \alpha|\left(\frac{r^{2}+1 / r^{2}}{r-1 / r}\right) .
$$

If $r=1+\varepsilon$, where $\varepsilon \ll 1$, we obtain:

$$
\Delta V_{g}=4 a \sqrt{-2 q}|\sin \alpha|\left(\frac{1}{r-1 / r}\right) .
$$

Comparing the expressions for $G$ and $\Delta V_{g}$, we have

$$
G=\gamma \Delta V_{g}
$$

where $\gamma$ is the parameter defined in the expression for breather solution (13).

Equation (26) clearly demonstrates the MI nature of the SR breathers. Namely, the MI growth rate of the SR breather coincides with the absolute value of the difference of group velocities.

The relation (26) is a remarkable result of physical significance. It holds not only for the infinite NLSE hierarchy [22] and the coupled NLSE-Maxwell-Bloch model [23] but also for the nonlinear model with a self-steepening described by the WKI system.

In the particular case of $V_{g 1}=-V_{g 2}$, we obtain $G=$ $2 \gamma\left|V_{g 1}\right|=2 \gamma\left|V_{g 2}\right|$ (namely, the growth rate of each quasi-AB is the same as that for the SR breather). We note that this particular relation can be obtained using simple physical arguments related to MI growth [26]. Formal asymptotic analysis [27] also confirms it. As we can see, it is valid for a wider class of localized perturbations. 


\section{CONCLUSION}

In conclusion, we studied SR breathers in a nonlinear system governed by the CLLE with the term that explicitly takes into account the self-steepening effect on ultrashort pulses. We have shown that these solutions are tightly related to the modulation instability affected by the asymmetric evolution caused by the self-steepening effect. These phenomena have been shown both using the exact solutions and numerical simulations. Asymmetry appears in the evolution dynamics even when it is started with the symmetric initial conditions.

Despite this symmetry breaking, the corresponding MI nature of evolution is confirmed by showing that the MI growth rate driven by the SR breather coincides with the absolute value of the difference of group velocities. Our results provide a route to the experimental verification of symmetry-broken MI dynamics in nonlinear systems with self-steepening, such as in optical frequency-doubling crystals and in magnetized plasmas.

\section{ACKNOWLEDGMENTS}

The work was conceived when C.L. visited Optical Sciences Group, ANU. This work is supported by NSFC (Grants No. 11705145, No. 11434013, and No. 11425522), the Major Basic Research Program of Natural Science of Shaanxi Province (Grants No. 2017KCT-12 and No. 2017ZDJC-32), Natural Science Basic Research Plan in Shaanxi Province of China (Grant No. 2018JQ1003), and Australian Research Council (Discovery Project No. DP150102057).

\section{APPENDIX A: FUNDAMENTAL (FIRST-ORDER) BREATHER SOLUTIONS}

As follows from Eq. (10), the quadratic spectral parameter corresponding to the fundamental (first-order) breather solution is

$$
\lambda_{1}^{2}=i \frac{a}{4}\left(\xi_{1}+\frac{1}{\xi_{1}}\right) \sqrt{-2 q}-\frac{1}{4}\left(a^{2}+2 q\right),
$$

where $\xi_{1}=r_{1} e^{i \alpha_{1}}$. The corresponding eigenfunctions $\left(R_{1}, S_{1}\right)$ of the Lax pair (6) are

$$
\begin{gathered}
R_{1}=\left[\left(\beta_{1}+f_{1}\right) e^{\chi_{1}}+i a \lambda_{1} e^{-\chi_{1}}\right] e^{\frac{i \theta}{2}}, \\
S_{1}=\left[\left(\beta_{1}+f_{1}\right) e^{-\chi_{1}}+i a \lambda_{1} e^{\chi_{1}}\right] e^{-\frac{i \theta}{2}},
\end{gathered}
$$

where $\beta_{1}=\beta\left(\lambda_{1}^{2}\right), f_{1}=f\left(\lambda_{1}^{2}\right)$, and

$$
\chi_{1}=\tau_{1}\left(t-\rho_{1} z\right)+\frac{i \theta_{1}}{2},
$$

with $\tau_{1}=i f_{1}$, and $\rho_{1}=\rho\left(\lambda_{1}^{2}\right)$.

The fundamental breather solution can be obtained through a Darboux transformation [37]. Namely,

$$
\psi=\frac{\psi_{0}\left(\lambda_{1}\left|S_{1}\right|^{2}+\lambda_{1}^{*}\left|R_{1}\right|^{2}\right)}{\lambda_{1}\left|R_{1}\right|^{2}+\lambda_{1}^{*}\left|S_{1}\right|^{2}}-\frac{2 i\left(\lambda_{1}^{2}-\lambda_{1}^{2 *}\right) R_{1} S_{1}^{*}}{\lambda_{1}\left|R_{1}\right|^{2}+\lambda_{1}^{*}\left|S_{1}\right|^{2}},
$$

where $*$ denotes the complex conjugate, $\lambda_{1}=\sqrt{\lambda_{1}^{2}}$. The simplified form of Eq.(A4) is given by Eq. (13).

\section{APPENDIX B: SECOND-ORDER BREATHER SOLUTIONS}

The exact SR breather solution of the CLLE (1) can be obtained in a second step of a Darboux transformation from the fundamental breather solution Eq. (A4) or Eq. (13). The technique for finding the multibreathers has been first developed in Ref. [46] and applied for the limiting case of the higher-order Akhmediev-Peregrine rogue waves [7]. Below we present the general determinant form of the two breather solution. In the particular case, when $r_{1}=r_{2}=1+\varepsilon$, and $\alpha_{1}=-\alpha_{2}=\alpha$, the solution describes the SR breather dynamics presented in Sec. IV.

The second quadratic spectral parameter involved in the solution is

$$
\lambda_{2}^{2}=i \frac{a}{4}\left(\xi_{2}+\frac{1}{\xi_{2}}\right) \sqrt{-2 q}-\frac{1}{4}\left(a^{2}+2 q\right), \quad \xi_{2}=r_{2} e^{i \alpha_{2}}
$$

The corresponding eigenfunctions $\left(R_{2}, S_{2}\right)$ of the Lax pair (6) are

$$
\begin{aligned}
& R_{2}=\left[\left(\beta_{2}+f_{2}\right) e^{\chi_{2}}+i a \lambda_{2} e^{-\chi_{2}}\right] e^{\frac{i \theta}{2}}, \\
& S_{2}=\left[\left(\beta_{2}+f_{2}\right) e^{-\chi_{2}}+i a \lambda_{2} e^{\chi_{2}}\right] e^{-\frac{i \theta}{2}},
\end{aligned}
$$

where $\beta_{2}=\beta\left(\lambda_{2}^{2}\right), f_{2}=f\left(\lambda_{2}^{2}\right)$, and

$$
\chi_{2}=\tau_{2}\left(t-\rho_{2} z\right)+\frac{i \theta_{2}}{2}
$$

with $\tau_{2}=i f_{2}$ and $\rho_{2}=\rho\left(\lambda_{2}^{2}\right)$. Then the two-breather solution can be written in the following determinant form:

$$
\psi=\frac{1}{\Omega_{21}}\left(\psi_{0} \Omega_{11}-2 i \Omega_{12}\right),
$$

where

$$
\begin{aligned}
\Omega_{21} & =\left|\begin{array}{cccc}
\lambda_{1}^{3} R_{1} & \lambda_{1}^{2} S_{1} & \lambda_{1} R_{1} & S_{1} \\
-\left(\lambda_{1}^{*}\right)^{3} S_{1}^{*} & \left(\lambda_{1}^{*}\right)^{2} R_{1}^{*} & -\lambda_{1}^{*} S_{1}^{*} & R_{1}^{*} \\
\lambda_{2}^{3} R_{2} & \lambda_{2}^{2} S_{2} & \lambda_{2} R_{2} & S_{2} \\
-\left(\lambda_{2}^{*}\right)^{3} S_{2}^{*} & \left(\lambda_{2}^{*}\right)^{2} R_{2}^{*} & -\lambda_{2}^{*} S_{2}^{*} & R_{2}^{*}
\end{array}\right|, \\
\Omega_{11} & =\left|\begin{array}{cccc}
\lambda_{1}^{3} S_{1} & \lambda_{1}^{2} R_{1} & \lambda_{1} S_{1} & R_{1} \\
-\left(\lambda_{1}^{*}\right)^{3} R_{1}^{*} & \left(\lambda_{1}^{*}\right)^{2} S_{1}^{*} & -\lambda_{1}^{*} R_{1}^{*} & S_{1}^{*} \\
\lambda_{2}^{3} S_{2} & \lambda_{2}^{2} R_{2} & \lambda_{2} S_{2} & R_{2} \\
-\left(\lambda_{2}^{*}\right)^{3} R_{2}^{*} & \left(\lambda_{2}^{*}\right)^{2} S_{2}^{*} & -\lambda_{2}^{*} R_{2}^{*} & S_{2}^{*}
\end{array}\right|, \\
\Omega_{12} & =\left|\begin{array}{cccc}
\lambda_{1}^{4} R_{1} & \lambda_{1}^{2} R_{1} & \lambda_{1} S_{1} & R_{1} \\
\left(\lambda_{1}^{*}\right)^{4} S_{1}^{*} & \left(\lambda_{1}^{*}\right)^{2} S_{1}^{*} & -\lambda_{1}^{*} R_{1}^{*} & S_{1}^{*} \\
\lambda_{2}^{4} R_{2} & \lambda_{2}^{2} R_{2} & \lambda_{2} S_{2} & R_{2} \\
\left(\lambda_{2}^{*}\right)^{4} S_{2}^{*} & \left(\lambda_{2}^{*}\right)^{2} S_{2}^{*} & -\lambda_{2}^{*} R_{2}^{*} & S_{2}^{*}
\end{array}\right| .
\end{aligned}
$$

The solution (B4) is general and allows us to consider the case of parallel breathers [14,55]. The condition for this is $V_{g 1}=V_{g 2}$. 
[1] J. M. Dudley, G. Genty, F. Dias, B. Kibler, and N. Akhmediev, Modulation instability, Akhmediev breathers and continuous wave supercontinuum generation, Opt. Express 17, 21497 (2009).

[2] B. Kibler, J. Fatome, C. Finot, G. Millot, F. Dias, G. Genty, N. Akhmediev, and J. M. Dudley, The Peregrine soliton in nonlinear fibre optics, Nat. Phys. 6, 790 (2010).

[3] M. Onorato, S. Residori, U. Bortolozzo, A. Montina, and F. T. Arecchi, Rogue waves and their generating mechanisms in different physical contexts, Phys. Rep. 528, 47 (2013).

[4] J. M. Dudley, F. Dias, M. Erkintalo, and G. Genty, Instabilities, breathers and rogue waves in optics, Nat. Photonics 8, 755 (2014).

[5] N. Akhmediev, B. Kibler, F. Baronio et al., Roadmap on optical rogue waves and extreme events, J. Optics 18, 063001 (2016).

[6] M. Onorato, S. Residori, and F. Baronio (eds.), Rogue and Shock Waves in Nonlinear Dispersive Media (Springer, Berlin, 2016).

[7] N. Akhmediev, A. Ankiewicz, and J. M. Soto-Crespo, Fundamental rogue waves and their superpositions in nonlinear integrable systems, in Nonlinear Guided Wave Optics: A Testbed for Extreme Waves, edited by S. Wabnitz (IOP Publishing, Bristol, 2017).

[8] M. Närhi, B. Wetzel, C. Billet, S. Toenger, T. Sylvestre, J.M. Merolla, R. Morandotti, F. Dias, G. Genty, and J. M. Dudley, Real-time measurements of spontaneous breathers and rogue wave events in optical fibre modulation instability, Nat. Commun. 7, 13675 (2016).

[9] A. Tikan, S. Bielawski, C. Szwaj, S. Randoux, and P. Suret, Single-shot measurement of phase and amplitude by using a heterodyne time-lens system and ultrafast digital timeholography, Nat. Photon. 12, 228 (2018).

[10] N. Akhmediev, A. Ankiewicz, and M. Taki, Waves that appear from nowhere and disappear without a trace, Phys. Lett. A 373, 675 (2009).

[11] N. Akhmediev, J. Soto-Crespo, and A. Ankiewicz, Extreme waves that appear from nowhere: On the nature of rogue waves, Phys. Lett. A 373, 2137 (2009).

[12] N. Akhmediev, J. Soto-Crespo, and A. Ankiewicz, How to excite a rogue wave, Phys. Rev. A 80, 043818 (2009).

[13] B. Frisquet, B. Kibler, and G. Millot, Collision of Akhmediev Breathers in Nonlinear Fiber Optics, Phys. Rev. X 3, 041032 (2013).

[14] G. Xu, A. Gelash, A. Chabchoub, V. Zakharov, and B. Kibler, Breather Wave Molecules, Phys. Rev. Lett. 122, 084101 (2019).

[15] C. Liu, Z. Y. Yang, W. L. Yang, and N. Akhmediev, Chessboardlike spatio-temporal interference patterns and their excitation, J. Opt. Soc. Am. B 36, 1294 (2019).

[16] V. E. Zakharov and A. A. Gelash, Nonlinear Stage of Modulation Instability, Phys. Rev. Lett. 111, 054101 (2013).

[17] A. A. Gelash and V. E. Zakharov, Superregular solitonic solutions: A novel scenario for the nonlinear stage of modulation instability, Nonlinearity 27, R1 (2014).

[18] B. Kibler, A. Chabchoub, A. Gelash, N. Akhmediev, and V. E. Zakharov, Superregular Breathers in Optics and Hydrodynamics: Omnipresent Modulation Instability Beyond Simple Periodicity, Phys. Rev. X 5, 041026 (2015).

[19] L. D. Faddeev and L. A. Takhtajan, Hamiltonian Methods in the Theory of Solitons (Springer, Heidelberg, 2007).

[20] J. H. Zhang, L. Wang, and C. Liu, Superregular breathers, characteristics of nonlinear stage of modulation instability induced by higher-order effects, Proc. R. Soc. A 473, 20160681 (2017).

[21] C. Liu, Y. Ren, Z. Y. Yang, and W. L. Yang, Superregular breathers in a complex modified Korteweg-de Vries system, Chaos 27, 083120 (2017).

[22] C. Liu, Z. Y. Yang, and W. L. Yang, Growth rate of modulation instability driven by superregular breathers, Chaos 28, 083110 (2018).

[23] Y. Ren, C. Liu, Z. Y. Yang, and W. L. Yang, Polariton superregular breathers in a resonant erbium-doped fiber, Phys. Rev. E 98, 062223 (2018).

[24] Y. Ren, X. Wang, C. Liu, Z. Y. Yang, and W. L. Yang, Characteristics of fundamental and superregular modes in a multiple self-induced transparency system, Commun. Nonlinear. Sci. Numer. Simulat. 63, 161 (2018).

[25] A. A. Gelash, Formation of rogue waves from the locally perturbed condensate, Phys. Rev. E 97, 022208 (2018).

[26] M. Conforti, S. Li, G. Biondini, and S. Trillo, Auto-modulation versus breathers in the nonlinear stage of modulational instability, Opt. Lett. 43, 5291 (2018).

[27] S. Trillo and M. Conforti, Quantitative approach to breather pair appearance in nonlinear modulational instability, Opt. Lett. 44, 4275 (2019).

[28] M. Wadati, K. Konno, and Y. H. Ichikawa, A generalization of the inverse scattering method, J. Phys. Soc. Jpn. 46, 1965 (1979).

[29] G. Agrawal, Nonlinear Fiber Optics, 5th ed. (Academic Press, San Diego, 2012).

[30] F. DeMartini, C. H. Townes, T. K. Gustafson, and P. L. Kelley, Self-steepening of light pulses, Phys. Rev. 164, 312 (1967); D. Anderson and M. Lisak, Nonlinear asymmetric self-phase modulation and self-steepening of pulses in long optical waveguldes, Phys. Rev. A 27, 1393 (1983).

[31] S. Chen, F. Baronio, J. M. Soto-Crespo, Y. Liu, and Ph. Grelu, Chirped Peregrine solitons in a class of cubic-quintic nonlinear Schrödinger equations, Phys. Rev. E 93, 062202 (2016).

[32] S. Chen, F. Baronio, J. M. Soto-Crespo, Ph. Grelu, and D. Mihalache, Versatile rogue waves in scalar, vector, and multidimensional nonlinear systems, J. Phys. A: Math. Theor. 50, 463001 (2017).

[33] F. Baronio, S. Chen, and, D. Mihalache, Two-color walking Peregrine solitary waves, Opt. Lett. 42, 3514 (2017).

[34] H. H. Chen, Y. C. Lee, and C. S. Liu, Integrability of nonlinear Hamiltonian systems by inverse scattering method, Phys. Scr. 20, 490 (1979).

[35] J. Moses, B. A. Malomed, and F. W. Wise, Self-steepening of ultrashort optical pulses without self-phase-modulation, Phys. Rev. A 76, 021802(R) (2007).

[36] J. Moses and F. W. Wise, Controllable Self-Steepening of Ultrashort Pulses in Quadratic Nonlinear Media, Phys. Rev. Lett. 97, 073903 (2006).

[37] Y. Zhang, L. Guo, J. He, and Z. Zhou, Darboux transformation of the second-type derivative nonlinear Schrödinger equation, Lett. Math. Phys. 105, 853 (2015).

[38] H. Triki, M. M. Babatin, and A. Biswas, Chirped bright solitons for Chen-Lee-Liu equation in optical fibers and PCF, Optik 149, 300 (2017).

[39] M. Conforti, F. Baronio, and S. Trillo, Dispersive shock waves in phase-mismatched second-harmonic generation, Opt. Lett. 37, 1082 (2012). 
[40] D. J. Kaup and A. C. Newell, An exact solution for a derivative nonlinear Schrödinger equation, J. Math. Phys. 19, 798 (1978).

[41] K. Mio, T. Ogino, K. Minami, and S. Takeda, Modifed nonlinear Schrödinger equation for Alfvén waves propagating along the magnetic field in cold plasmas, J. Phys. Soc. Japan. 41, 265 (1976).

[42] A. S. Fokas, On a class of physically important integrable equations, Physica D 87, 145 (1995); J. Lenells, Exactly solvable model for nonlinear pulse propagation in optical fibers, Stud. Appl. Math. 123, 215 (2009).

[43] A. Kundu, Landau-Lifshitz and higher-order nonlinear systems gauge generated from nonlinear Schrödinger-type equations, J. Math. Phys. 25, 3433 (1984).

[44] J. Zhang, W. Liu, D. Qiu, Y. Zhang, K. Porsezian, and J. He, Rogue wave solutions of a higher-order Chen-Lee-Liu equation, Phys. Scripta 90, 055207 (2015).

[45] M. Wadati and K. Sogo, Gauge transformations in soliton theory, J. Phys. Soc. Jpn. 52, 394 (1983).

[46] N. Akhmediev, V. I. Korneev, and N. V. Mitskevich, Nmodulation signals in a single-mode optical waveguide under nonlinear conditions, Sov. Phys. JETP 67, 89 (1988) [Zh. Exp. Teor. Fiz. 94, 159 (1988)].

[47] N. Akhmediev and V. I. Korneev, Modulation instability and periodic solutions of the nonlinear Schrödinger equation, Theor. Math. Phys. 69, 1089 (1986).
[48] E. A. Kuznetsov, Solitons in a parametrically unstable plasma, Sov. Phys. Dokl. 22, 575 (1977); Y. C. Ma, The perturbed plane wave solutions of the cubic nonlinear Schrödinger equation, Stud. Appl. Math. 60, 43 (1979).

[49] D. H. Peregrine, Water waves, nonlinear Schrödinger equations and their solutions, J. Aust. Math. Soc. Ser. B 25, 16 (1983).

[50] D. J. Kedziora, A. Ankiewicz, A. Chowdury, and N. Akhmediev, Integrable equations of the infinite nonlinear Schrödinger equation hierarchy, Chaos 25, 103114 (2015).

[51] M. Tajiri and Y. Watanabe, Breather solutions to the focusing nonlinear Schrödinger equation, Phys Rev. E 57, 3510 (1998).

[52] N. Akhmediev and A. Ankiewicz, Solitons: Nolinear Pulses and Beams (Chapman \& Hall, London, 1997).

[53] G. I. Stegeman and M. Segev, Optical spatial solitons and their interactions: Universality and diversity, Science 286, 1518 (1999).

[54] J. M. Dudley, F. Gutty, S. Pitois, and G. Millot, Complete characterization of $\mathrm{THz}$ pulse trains generated from nonlinear processes in optical fibers, IEEE J. Quant. Electron. 37, 587 (2001).

[55] S. Li and G. Biondini, Soliton interactions and degenerate soliton complexes for the focusing nonlinear Schrödinger equation with nonzero background, Eur. Phys. J. Plus 133, 400 (2018). 